\title{
Minitrack Introduction: Decision Support for Smart City and Digital Services
}

\author{
Wei Xu \\ School of Information \\ Renmin University of China \\ Beijing, 100872, China \\ weixu@ruc.edu.cn
}

\author{
Jian Ma \\ Department of Information \\ Systems \\ City University of Hong Kong \\ Hongkong, China \\ isjian@cityu.edu.hk
}

\author{
Jianshan Sun \\ School of Management \\ Hefei University of Technology \\ Anhui, 230009, China \\ sunjs9413@gmail.com
}

Developing smart city and enhancing digital services are the critical important to urbanization process for improving the effectiveness and efficiency of traditional cities. With the massive applications of Internet of things (IoT), mobile networks, and social networks, unprecedentedly large amount of various heterogeneous data can be gathered and processed in terms of advanced analytics to support smart applications and digital services. Furthermore, decision support tools and big data analytics can be employed to speed up the whole process.

This minitrack addresses issues that focus on the applications of various decision support tools, such as decision analysis, soft computing, and big data analytics, to develop smart city applications and digital services. We also encourage papers to report on system level research and case studies related to smart city and digital society.

In this minitrack, four papers have been accepted. The first paper titled "The development of a smart map for minimum 'exertion' routing applications" designs an algorithm for topograph construction, and introduces a topographical network to minimize cost routing based on the exertion metric. The empirical results show the effectiveness of the proposed method. The second paper titled "Model design and implementation of enterprise credit information based on data mining" constructs a corporate credit risk assessment model based on governmental data using logit regression. The experimental results show that the proposed model is an alterantive tool for corporate credit risk evaluation. The third paper titled "Investigating the relationship among characteristics of social commerce, consumers' trust and trust performance" investigates the characteristics of social commerce influencing consumers' trust and assesses the effects of trust on trust performance. The empirical analysis results show that all the characteristics of social commerce involved had significant effects on trust which will also positively influence trust performance and making purchase decisions. The fourth paper titled "Connecting researchers with companies for university-industry collaboration" proposed a social network based patent trading model by leveraging the benefits of social commerce in identifying potential customers, providing distinguished marketing strategy, and maintaining customer loyalty in comparison with traditional ecommerce to enhance the transaction trust with social presence. The empirical analysis shows that the proposed method has achieved the satisfactory results.

In summary, smart city and digital services have played a critical important role in real life. We believe that there are more theoretical contributions and novel applications in smart city and digital services in future. 